\title{
VARIANTe CAMBial en tallos de IPOMoEa (Convolvulaceae) NATIVAS de Argentina
}

\author{
NATALIA DELBÓN¹, AGUSTINA SÁNCHEZ y MARÍA TERESA COSA
}

\begin{abstract}
Summary: Cambial variant in stems of Ipomoea (Convolvulaceae) native from Argentine. Ipomoea is a diverse and complex genus, composed of herbaceous plants, woody lianas and vines, and a small group of shrubs and trees. It is noteworthy that all species studied previously presented different types of cambial variant in their stems, regardless of habit, except two taxa that showed normal secondary growth, a vine and a tree. Therefore, in this paper we study three vines natives from Argentina, Ipomoea cairica, I. purpurea and $I$. rubriflora, and $I$. carnea ssp. fistulosa, a tree with normal growth, in order to compare the development of their stems and provide useful information to elucidate their phylogenetic relationships. The four species studied showed the same ontogeny and anatomy of their stems with primary growth, which would support its narrow phylogenetic relationships. On the other hand, the vines had only two types of cambial variant, the lobed pattern and supernumerary cambium. We can infer that the type of secondary growth and cambial variant types would not be directly related to the habit; however, other wood features can be considered as the proportion of parenchymal tissue and characteristics of the vessels.
\end{abstract}

Key words: Ipomoea, vining species, cambial variant, cambium.

Resumen: Ipomoea es un género diversificado y complejo, compuesto por herbáceas, lianas y enredaderas leñosas, y un pequeño grupo de arbustos y árboles. Llama la atención que todas las especies estudiadas anteriormente presentaron diferentes tipos de variante cambial en sus tallos, independientemente del modo de vida, excepto dos taxones con crecimiento secundario normal, una liana y un árbol. Por ello, en el presente trabajo se planteó el estudio de tres enredaderas nativas de Argentina, Ipomoea cairica, I. purpurea e I. rubriflora, y de I. carnea ssp. fistulosa, árbol con crecimiento normal, con el fin de comparar el desarrollo de sus tallos y aportar datos de interés para dilucidar sus relaciones filogenéticas. Las cuatro especies estudiadas presentaron igual ontogenia y anatomía de sus tallos con crecimiento primario, lo que apoyaría sus estrechas relaciones filogenéticas. Por otro lado, las enredaderas presentaron sólo dos tipos de variante cambial, el patrón lobado y el de cámbium supernumerario. Se puede inferir que en Ipomoea el tipo de crecimiento secundario, así como los tipos de variante cambial, no estarían directamente relacionados con el hábito; sin embargo, otras características del leño pueden ponerse a consideración como la proporción de tejido parenquimático y características de los vasos.

Palabras clave: Ipomoea, enredaderas, variante cambial, cámbium.

\section{INTRODUCCIÓN}

Ipomoea L. (Convolvulaceae) es un género diversificado y complejo, compuesto por unas 600 especies (Austin, 1980); muchas de ellas utilizadas

1 Laboratorio de Morfología Vegetal. Instituto Multidisciplinario de Biología Vegetal (UNC-CONICET). Facultad de Ciencias Exactas, Físicas y Naturales. Universidad Nacional de Córdoba. en medicina popular, cultivadas como ornamentales y/o consideradas invasoras (Frey, 1995; Barboza et al., 2006; Shaltout et al., 2006).

En base a caracteres morfoanatómicos fue dividido en tres subgéneros y numerosas secciones y series (Austin, 1979, 1980; Austin \& Huáman, 1996); sin embargo, estas delimitaciones, tanto genérica como infragenéricas, no fueron sostenidas por los análisis filogenéticos realizados, por lo que se encuentran actualmente en discusión (Miller et al., 1999; Manos et al., 2001; Stefanovic et al., 2003). 
Sus especies son comúnmente herbáceas volubles o rastreras, en menor proporción se encuentran lianas y enredaderas leñosas, pero además, existe un pequeño grupo con porte erguido, desde arbustos hasta árboles (Austin, 1980). Frente a esta diversidad de hábitos, es de esperar que se presenten diferentes modificaciones estructurales en sus tallos, ya que, en general las especies con porte erguido exhiben un crecimiento secundario normal, considerado basal o ancestral; en tanto que las especies leñosas de trepadoras y lianas presentan diferentes tipos de variante cambial, los cuales serían caracteres derivados (McDonald, 1992; Carlquist, 2001, 2007; Angyalossy et al., 2011; Schnitzer, 2015).

Sin embargo, en el género Ipomoea, todas las especies estudiadas hasta el momento presentaron algún tipo de variante cambial o patrón de crecimiento secundario anómalo, independientemente del modo de vida (Lowell \& Lucansky, 1986, 1990; Carlquist \& Hanson, 1991; McDonald, 1992; Ceja-Romero \& Pérez-Olvera, 2010; Terrazas et al., 2011; Rajput et al., 2014). Solamente existen dos excepciones, en las cuales se observó crecimiento secundario normal, $I$. carnea ssp. carnea e I. carnea ssp. fistulosa, siendo la primera una liana y la última un árbol (McDonald, 1992; Frey, 1995). Para este género entonces, se planteó que las características basales serían el hábito trepador con variante cambial, mientras que el porte erguido sería derivado (McDonald, 1992; Manos et al., 2001).

A pesar de los numerosos estudios en este género, escasos son los trabajos realizados sobre la ontogenia de tallos (Lowell \& Lucansky, 1986, 1990; Rajput et al., 2008; Terraza et al., 2011), fundamentales para comprender el desarrollo de los distintos tipos de variante cambial. Por ello, se planteó el estudio de la ontogenia, anatomía y desarrollo de enredaderas nativas de Argentina, y de I. carnea ssp. fistulosa, árbol con crecimiento normal nativo de México. Se pretende analizar y comparar los diferentes tipos de crecimientos y aportar datos de interés para dilucidar las relaciones filogenéticas de este complejo género.

\section{Materiales y Métodos}

Las especies estudiadas provienen de Argentina, prov. de Córdoba, los ejemplares de herbario se encuentran depositados en el Museo Botánico de Córdoba (CORD). Las tres enredaderas fueron recolectadas en la ciudad de Río Ceballos, Reserva Hídrica La Quebrada, ellas son: Ipomoea cairica (L.) Swett (25-I-2015, Cosa 414), I. purpurea (L.) Roth (25-I-2015, Cosa 415) e I. rubriflora O'Donell (25-I2015, Cosa 416). Ipomoea carnea Jacq. ssp. fistulosa (Mart. ex Choisy) fue recolectada en la ciudad de Córdoba (20-IV-2015, Cosa 417).

Con el material fresco se realizaron cortes transversales a mano alzada por tallos en diferentes estadios de desarrollo, desde el ápice caulinar hasta tallos con crecimiento secundario, los cuales fueron sometidos a pruebas histoquímicas, se utilizó Lugol (IIK) para revelar almidón y Sudan IV para lípidos. Con material fijado en FAA se confeccionaron iguales cortes que fueron teñidos con Azul AstralFucsina Básica y montados con glicerina 50\%. Las fotomicrografías se tomaron con microscopio Zeiss Axiophot y cámara digital Leica DFC300FX.

\section{Resultados}

\section{Crecimiento primario}

Las cuatro especies estudiadas presentaron igual ontogenia y anatomía de sus tallos con estructura primaria (Fig. 1 A-B, 2 A-B). La epidermis fue uniestratificada con cutícula fina. La corteza presentó 3-5 estratos de colénquima y luego parénquima compacto, siendo este más desarrollado en I. carnea ssp. fistulosa. En el parénquima cortical y medular se observaron gran cantidad de laticíferos, que contendrían sustancias lipídicas, ya que reaccionaron con Sudán IV. Por su parte, el parénquima medular contuvo abundante almidón, revelado con Lugol.

El sistema vascular presentó dos etapas de desarrollo. En cortes por ápice caulinar (Fig. 1 A), el procámbium se observó como un anillo continuo, del que derivaron pequeños grupos de protofloema centrífugamente y protoxilema centrípetamente; este último formado por vasos de diámetro reducido. El floema interno se diferenció a partir de células parenquimáticas del protoxilema, conformando así haces bicolaterales.

En una etapa ontogenética posterior (Fig. 1 B y 2 A-B) el procámbium formó un anillo continuo de metafloema centrífugamente y uno de mayor espesor de metaxilema centrípetamente. Este último se diferencia notablemente del protoxilema por encontrarse integrado solo por traqueidas. El floema interno se desarrolla pero se mantiene en grupos. 




Fig. 1. Corte transversal por tallo con diferentes estadios de desarrollo de Ipomoea carnea ssp. fistulosa. A: Sector del tallo con incipiente desarrollo del sistema vascular, formando hacecillos bicolaterales. B: Tallo con crecimiento primario, protoxilema y metaxilema diferenciados. C: Tallo con crecimiento secundario, xilema formado por abundantes traqueidas. D: Detalle de vasos agrupados linealmente. E: Detalle de floema y corteza con laticíferos. F: Almidón en radios y médula revelado con Lugol (IIK), y drusas en corteza y médula. Abreviaturas: ca: cámbium, co: corteza, ep: epidermis, f: fibras, fin: floema interno, fp: floema primario, fs: floema secundario, m: médula, mx: metaxilema, pc: procámbium; pe: peridermis, pf: protofloema, px: protoxilema, r: radio, tr: traqueida, v: vaso, xp: xilema primario, xs: xilema secundario, flecha: almidón, punta de flecha: drusas, asterisco: laticífero. Escala: $300 \mu \mathrm{m}$.

\section{Crecimiento secundario normal}

En la especie de hábito arbóreo, I. carnea ssp. fistulosa, el cámbium presentó una actividad normal, formando de manera continua y completa xilema centrípetamente y floema centrífugamente (Fig. 1 C). El xilema secundario tuvo gran desarrollo, con mayor proporción de traqueidas.
Los vasos fueron escasos, agrupados linealmente y en algunos casos obstruidos por sustancias lipídicas; no presentaron tílides. Los radios xilemáticos fueron numerosos pero uniseriados y se observó escaso parénquima axial paratraqueal (Fig. 1 D). El floema secundario presentó menor desarrollo, destacándose radios floemáticos uniseriados con 


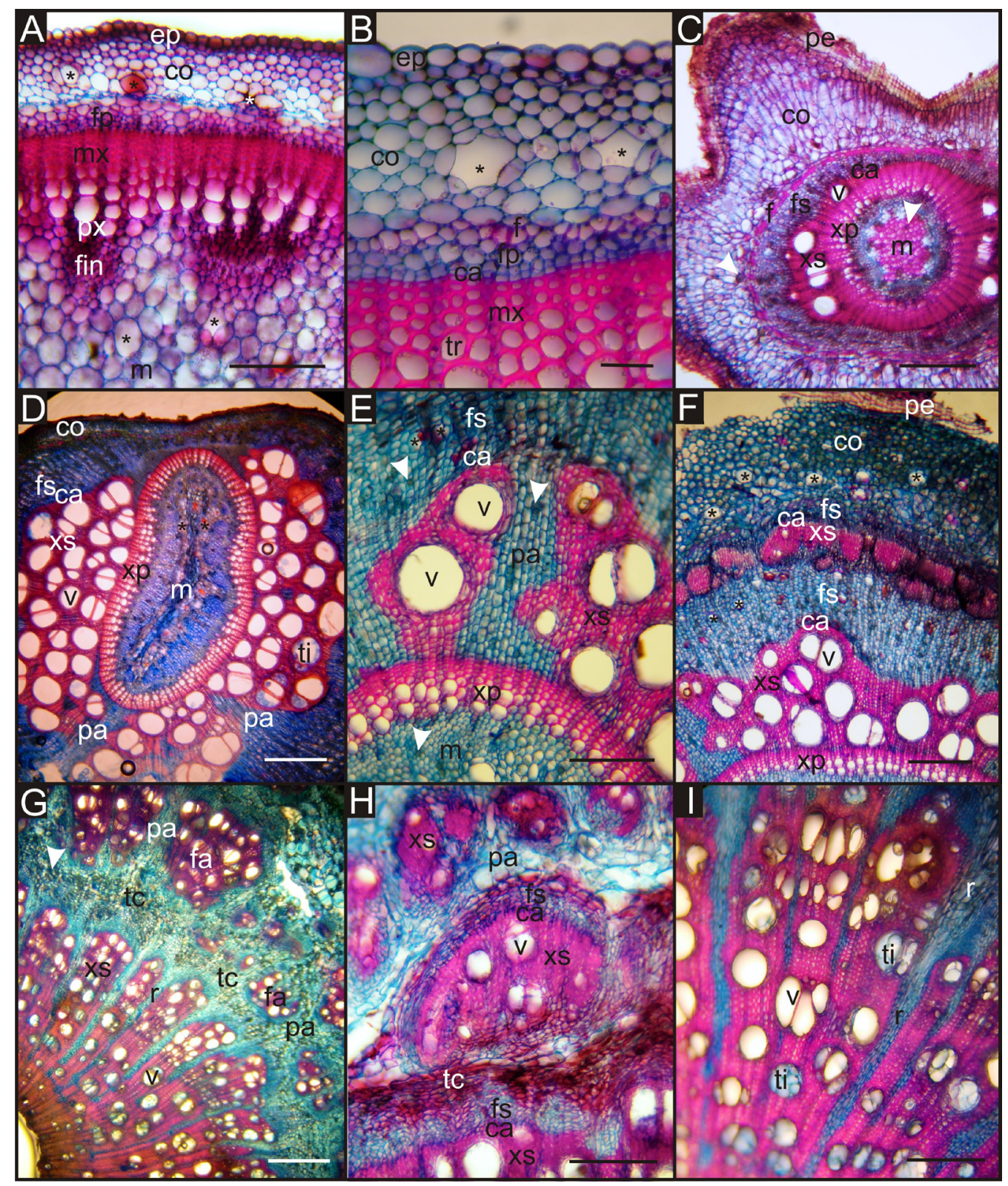

Fig. 2. Corte transversal por tallo con diferentes estadios de desarrollo de I. rubriflora (A, B, E, F), I. cairica (C) e I. purpurea (D, G, H, I). A: Crecimiento primario, metaxilema y protoxilema diferenciados. B: Detalle de corteza con laticíferos. C: Primera variante cambial, comienzo del crecimiento secundario con patrón lobado. D: Patrón lobado desarrollado. E: Detalle destacando una banda de tejido parenquimatico. F: Segunda variante cambial, comienzo del desarrollo de cámbium supernumerario. G: Anillos concéntricos discontinuos. H: Fascículos con escaso floema y mayor proporción de xilema, separados entre sí por tejido parenquimático. I: zona del xilema con patrón lobado, desarrollo de radios xilemáticos multiseriados y vasos con tílides. Abreviaturas: ca: cámbium, co: corteza, ep: epidermis, f: fibras, fa: fascículo, fin: floema interno, $\mathrm{fp}$ : floema primario, fs: floema secundario, m: médula, mx: metaxilema, pa: parénquima, pe: peridermis, px: protoxilema, r: radio, tc: tejido conjuntivo, ti: tílide, tr: traqueidas, v: vaso, xp: xilema primario, xs: xilema secundario, punta de flecha: drusas, asteriscos: laticífero. Escalas: B: $50 \mu \mathrm{m} ; \mathrm{A}$ y F: $200 \mu \mathrm{m} ; \mathrm{C}, \mathrm{E}, \mathrm{H}-\mathrm{I}: 300$ $\mu \mathrm{m}$; D y G: $500 \mu \mathrm{m}$. 


\section{N. Delbón et al. - Variante cambial en tallos de Ipomoea nativas de Argentina}

drusas. Se desarrollaron externamente pequeños grupos de fibras (Fig. $1 \mathrm{E}$ ).

En corteza y médula los laticíferos se mantienen, y aparecen drusas (Fig. 1 E-F). El almidón se evidenció en abundancia en los radios xilemáticos y en menor proporción en la médula (Fig. $1 \mathrm{~F}$ ); cabe destacar que, a medida que avanza el desarrollo del tallo, el almidón medular se consume y las células degeneran, por lo que el tallo queda hueco.

\section{Variante cambial}

Las tres especies de hábito trepador presentaron dos patrones diferentes de crecimiento secundario anómalo o variante cambial. El patrón lobado es la primara anomalía que se produce, mientras que el patrón de cámbium supernumerario o sucesivos aparece en estadios avanzados del desarrollo del leño.

En el patrón lobado el cámbium original presentó una actividad normal pero diferencial, es decir, originó normalmente xilema centrípetamente y floema centrífugamente, pero con zonas de mucha producción de tejidos y otras zonas con escasa o nula producción (Fig. 2C). En consecuencia no se forman anillos continuos, sino lóbulos, lo que le confiere un aspecto asimétrico e irregular (Fig. 2 D). El floema estuvo formado por elementos conductores, parénquima y radios floemáticos uniseriados, y presentó laticíferos y drusas. En el xilema se destacan vasos de gran diámetro, mayormente solitarios, o en grupos de 2 o 3, con tílides; los rodearon unas pocas células del parénquima axial, fibras, traqueidas y radios xilemáticos uniseriados. Además, en el xilema se observaron bandas radiales de células parenquimáticas con drusas (Fig. 2 E).

El siguiente estadio se caracterizó por la aparición de cámbium supernumerario o sucesivos, los cuales formaron de 2 a 4 anillos concéntricos de xilema y floema, separados por abundante tejido conjuntivo parenquimático (Fig. 2 F, G). Cada cámbium se desarrolló a partir de células parenquimáticas externas del floema secundario que lo precede, y fue discontinuo por lo que formó haces o fascículos de diferentes tamaños. Estos presentaron escaso floema centrífugo y mayor proporción de xilema centrípeto, separados entre sí por tejido parenquimático (Fig. $2 \mathrm{G}, \mathrm{H}$ ). Por su parte, en la zona interna, donde se encuentra el xilema secundario con patrón lobado, se desarrollan radios xilemáticos multiseriados y los vasos se obliteran o presentaron mayor proporción de tílides (Fig. 2 I). El almidón y las drusas se localizaron en los radios xilemáticos y floemáticos, y en el tejido conjuntivo. El parénquima medular colapsa y desaparece dejando los tallos huecos (Fig. 2 G).

\section{Discusión}

Pocas son las especies en las que se han estudiado en detalle las características de los tallos primarios y su ontogenia. Se destacan los estudios de ápices caulinares en I. hederiflora L. e I. quamoclit L. (Lowell \& Lucansky, 1986; 1990), y de plántulas en I. batatas (L.) Lam. (Hayward, 1932), I. aquatica Forssk. (Devi et al., 1990) e I. arborescens (Humb. \& Bonpl. ex Willd.) G. Don (Terrazas et al., 2011); en tanto, para $I$. purpurea se analizó la anatomía del tallo primario, sin su ontogenia, en relación a la fuerza utilizada para trepar (Scher et al., 2001). En todas ellas así como en las estudiadas en el presente trabajo, se observaron dos etapas ontogenéticas e iguales características anatómicas. Esta gran similitud apoyaría las estrechas relaciones filogenéticas entre estas especies, afirmando su permanencia en el género (Miller et al., 1999; Manos et al., 2001; Stefanovic et al., 2003); además, estaría en concordancia con la hipótesis de que el modo de vida autosostenido y con crecimiento secundario normal sería derivado, a partir de especies de lianas y enredaderas con variante cambial (McDonald, 1992; Angyalossy et al., 2011).

Con respecto al crecimiento secundario, $I$. carnea ssp. fistulosa mostró crecimiento secundario normal producido por el único cámbium original, que presentó una actividad continua. Esto, así como las características del leño, habían sido reportadas con anterioridad para esta especie y para I. carnea ssp. carnea, pero sin analizar su ontogenia (McDonald, 1992).

En cambio, en las tres especies de enredaderas aquí estudiadas se observaron dos tipos de variante cambial, el patrón lobado y el de cámbium supernumerario. El patrón lobado es la primara anomalía que se observa y se produce por una actividad diferencial del cámbium, con zonas de mucha producción de tejidos y otras nula. Estas observaciones sólo coinciden con lo observado en I. hederiflora e I. quamoclit en las 
que se observaron dos lóbulos opuestos (Lowell \& Lucansky, 1986; 1990), mientras que en las especies aquí estudiadas fueron dos o más. Esta asimetría en el sistema vascular de los tallos estaría asociada con el soporte sobre el que se apoyan para crecer (Lowell \& Lucansky, 1990; Carlquist, 2007; Angyalossy et al., 2011). El patrón de cámbium supernumerario aparece en estadios avanzados del desarrollo del leño. Estos forman numerosos anillos sucesivos de xilema, floema y tejido conjuntivo de células parenquimáticas, siendo estos anillos discontinuos, formando haces o fascículos. Este tipo de crecimiento anómalo es el más frecuente en el género (Metcalfe \& Chalk, 1950), y fue reportado en especies de trepadoras anuales (Lowell \& Lucansky, 1986; Rajput et al., 2008), y en enredaderas, árboles $\mathrm{y}$ arbustos de la serie Arborescentes, aunque en estas sólo se analizaron los leños de plantas adultas (McDonald, 1992; Ceja-Romero \& Pérez-Olvera, 2010). Nuestras observaciones no coinciden con lo reportado anteriormente por Chen et al. (2007), ya que ellos indicaron que I. cairica no presenta crecimiento secundario desarrollado.

Por su parte, en otras especies de Ipomoea se han reportado diferentes patrones de crecimiento, o variante cambial, que no fueron observadas en las especies estudiadas. Por ejemplo, en el patrón foraminado se forman pequeñas lagunas de floema incluidas en el xilema, a partir de cámbiumes intraxilares, (Lowell \& Lucansky, 1986,1990; Rajput et al., 2008, 2013). Además se ha reportado el desarrollo de cámbiumes con orientación invertida, que originan xilema centrífugamente y floema centrípetamente (Rajput et al., 2008; Terrazas et al., 2011). La aparición de cámbiumes internos entre el xilema primario y el floema interno, y que producen haces vascular invertidos (Lowell \& Lucansky, 1990; Rajput et al., 2008; Patil et al., 2009). Finalmente, el desarrollo de cámbiumes en los radios xilemáticos con capacidad de formar xilema y floema secundarios fue reportado solo en I. obscura (L.) Ker Gawl. y sería el único en la familia (Rajput et al., 2013).

Frente a esta heterogeneidad, se puede inferir que en Ipomoea el tipo de crecimiento secundario, normal o anómalo, así como los tipos de variante cambial, no estarían directamente relacionados con el hábito; sin embargo, otras características del leño pueden ponerse a consideración.

La gran proporción de tejido parenquimático que se observó en las tres especies de trepadoras estudiadas sería beneficioso ya que le confiere a los tallos mayor flexibilidad y fuerza tensil para enredarse y crecer. En cambio, en I. carnea spp. fistulosa, el parénquima es escaso y abunda el tejido esclerenquimático, lo cual estaría en concordancia con la necesidad de autosostenerse (Carlquist \& Hanson 1991; Carlquist, 2001; Gutiérrez et al., 2009; Angyalossy et al., 2011; Schnitzer, 2015). Iguales observaciones fueron reportadas para otras dos especies de arbustos, I. chilopsidis Standl. e I. rzedowskii E. Carranza, Zamudio \& Murguía, en los que a pesar de presentar cámbium supernumerario, el tejido conjuntivo parenquimático no se encuentra desarrollado y los anillos concéntricos son continuos (Ceja-Romero \& Pérez-Olvera, 2010).

Además, en las especies estudiadas se pudo evidenciar mediante pruebas histoquímicas la presencia de abundante almidón; de este modo, los tallos poseen la capacidad de reservar agua y nutrientes, los cuales pueden ser utilizados para sobrellevar los períodos de sequía (Carlquist, 2001; Angyalossy et al., 2011).

Sumado a esto, en integrantes Mexicanos de la serie Arborescentes de Ipomoea se reportaron diferencias cuantitativas del leño, las cuales podrían relacionarse con el hábito de los taxas; así, las lianas y arbustos resultaron diferente a los árboles en cuanto a tamaño y frecuencia de vasos y proporción de parénquima y fibras (Ceja-Romero \& Pérez-Olvera, 2010). Iguales conclusiones fueron reportadas para otras familias y géneros que presentan especies de lianas (Gutiérrez et al., 2009; Angyalossy et al., 2011; Schnitzer, 2015), por lo que se plantea la necesidad de ampliar los estudios cuantitativos del leño en especies Argentinas.

\section{Conclusiones}

Las cuatro especies estudiadas, así como las reportadas anteriormente, presentaron igual ontogenia y anatomía de sus tallos primario, lo que apoyaría sus estrechas relaciones filogenéticas. Por otro lado, I. carnea ssp. fistulosa mostró un crecimiento secundario normal producido por un único cámbium con actividad continua, en tanto que las enredaderas presentaron sólo dos tipos de variante cambial, el patrón lobado y el de cámbium supernumerario, lo cual difiere con lo reportado en otras especies. Se puede 


\section{N. Delbón et al. - Variante cambial en tallos de Ipomoea nativas de Argentina}

inferir que en Ipomoea el tipo de crecimiento secundario, así como los tipos de variante cambial, no estarían directamente relacionados con el hábito; sin embargo, otras características del leño pueden ponerse a consideración como la proporción de tejido parenquimático y características de los vasos.

\section{Agradecimientos}

Agradecemos al Dr. Franco Chiarini por la identificación de las especies y al financiamiento de de la Secretaría de Ciencia y Técnica, Universidad Nacional de Córdoba (SECYT-UNC) y del Consejo Nacional de Investigaciones Científicas y Técnicas (CONICET). ND es becaria posdoctoral de CONICET.

\section{Biblografía}

ANGYALOSSY, A, G. ANGELES, M. PACE, A. LIMA, C. DIAS-LEME, L. LOHMANN \& C. MADEROVEGA. 2011. An overview on the anatomy, development and evolution of the vascular system of lianas. Plant Ecol. Divers. 5: 167-182.

AUSTIN, D. 1979. An infrageneric classification for Ipomoea (Convolvulaceae). Taxon 28: 359-361.

AUSTIN, D. 1980. Additional comments on infrageneric taxa in Ipomoea (Convolvulaceae). Taxon 29: 501502.

AUSTIN, D. \& Z. HUÁMAN. 1996. A sinopsis of Ipomoea (Convolvulaceae) in the Americas. Taxon 45: 3-38.

BARBOZA, G., J. CANTERO, C. NUÑEZ \& L. ARIZA ESPINAR. 2006. Flora medicinal de la Provincia de Córdoba (Argentina). Museo Botánico de Córdoba, Argentina.

CARLQUIST, S. 2001. Comparative wood anatomy: Systematic, ecological and evolutionary aspects of dicotyledon wood. Springer, Berlin, Heidelberg.

CARLQUIST, S. 2007. Successive cambia revisited: Ontogeny, histology, diversity, and functional significance. J. Torrey Bot. Soc. 134: 301-332.

CARLQUIST, S. \& M. HANSON. 1991. Wood and stem anatomy of Convolvulaceae: a survey. Aliso 13: 5194.

CEJA-ROMERO, J. \& C. PÉREZ-OLVERA. 2010. Anatomía de la madera de las especies arbóreas de Ipomoea (Convolvulaceae). Madera y Bosques 16: 61-73.

CHEN, D., Y. DING, R. MA, H. ZHU \& A. LU. 2007. Anatomical structure of stem of Ipomoea cairica (Convolvulaceae). Acta Bot. Yunnan. 2: 189-192.
DEVI, A., M. PARABIA \& M. REDDY. 1990. Morphological and anatomical studies of seedling of Ipomoea aquatica Forsk. Feddes Repert. 101: 391-394.

GUTIÉRREZ, M., R. MIGUEL-CHÁVEZ \& T. TERRAZAS. 2009. Xylem conductivity and anatomical traits in diverse lianas and small tree species from tropical forest of southwest México. Int. J. Bot. 5: 279-286.

FREY, R. 1995. Ipomoea carnea ssp. fistulosa (Martius ex Choisy) Austin: taxonomy, biology and ecology reviewed and inquired. Trop. Ecol. 36: 21-48.

HAYWARD, E. 1932. The seedling anatomy of Ipomoea batatas. Bot. Gaz. 93: 400-420.

LOWELL, C. \& T. LUCANSKY. 1986. Vegetative anatomy and morphology of Ipomoea hederifolia (Convolvulaceae). Bull. Torrey Bot. Club. 113: 382397.

LOWELL, C. \& T. LUCANSKY. 1990. Vegetative anatomy and morphology of Ipomoea quamoclit (Convolvulaceae). Bull. Torrey Bot. Club. 117: 232246.

MANOS, P., R. MILLER \& P. WILKIN. 2001. Phylogenetic analysis of Ipomoea, Argyreia, Stictocardia, and Turbina. Suggest a Generalized Model of Morphological Evolution in Morning Glories. Syst. Bot. 26:585-602.

MCDONALD, J. 1992. Evolutionary implications of typical and anomalous secondary growth in arborescent Ipomoea (Convolvulaceae). Bull. Torrey Bot. Club.119: 262-267.

METCALFE, C. \& L. CHALK. 1950. Anatomy of the dicotyledons. Leaves, stem, and wood in relation to taxonomy with notes on economic uses. Vol. II, Clarendon Press, Oxford.

MILLER, R., M. RAUSHER \& P. MANOS. 1999. Phylogenetic Systematics of Ipomoea (Convolvulaceae) Based on ITS and Waxy Sequences. Syst. Bot. 24: 209-227.

PATIL, V., K. RAO \& K. RAJPUT. 2009. Development of intraxylary phloem and internal cámbium in Ipomoea hederifolia (Convolvulaceae). J. Torrey Bot. Soc. 136: 423-432.

RAJPUT, K., B. CHAUDHARY \& V. PATIL. 2014. Development of successive cambia and structure of secondary xylem of Ipomoea obscura (Convolvulaceae). Polish Bot. J. 59: 55-61.

RAJPUT, K., V. PATIL \& K. RAO. 2013. Wood anatomy and the development of interxylary phloem of Ipomoea hederifolia Linn. (Convolvulaceae). J. Plant. Growth Regul. 32: 654-662.

RAJPUT, K., V. RAOLE \& D. GANDHI. 2008. Radial secondary growth and formation of successive cambia and their products in Ipomoea hederifolia L. (Convolvulaceae). Bot. J. Linn. Soc. 158: 30-40. 
SCHNITZER, S., F. BONGERS, R. BURNHAM \& F. PUTZ. 2015. Ecology of lianas. Wiley-Blackwell Publishing, Oxford.

SCHER, J., N. HOLBROOK \& W. SILK. 2001. Temporal and spatial patterns of twining force and lignification in stems of Ipomoea purpurea. Planta 213: 192-198.

SHALTOUT, K., Y. AL-SODANY \& E. EID. 2006. The biology of Egyptian woody perennials 2. Ipomoea carnea Jacq. Ass. Univ. Bull. Environ. Res. 9: 75-91. STEFANOVIC, S., D. AUSTIN \& R. OLMSTEAD. 2003. Classification of Convolvulaceae: a phylogenetic approach. Syst. Bot. 28: 791-806.
TERRAZAS, T., S. AGUILAR-RODRÍGUEZ \& C. OJANGUREN 2011. Development of successive cambia, cambial activity, and their relationship to physiological traits in Ipomoea arborescens (Convolvulaceae) seedlings. Amer. J. Bot. 98: 765774.

Recibido el 15 de abril de 2016, aceptado el 30 de junio de 2016. 